\title{
The Phase-I Trigger Readout Electronics Upgrade of the ATLAS Liquid Argon Calorimeters
}

\author{
Ryunosuke Iguchi* \\ on behalf on the ATLAS Liquid Argon Calorimeter Group \\ University of Tokyo \\ E-mail: ryunosuke.iguchi@cern.ch
}

Electronics developments are pursued for the trigger readout of the ATLAS Liquid-Argon Calorimeter towards the Phase-I upgrade scheduled in the LHC shut-down period of 2019-2020. Trigger signals with higher spatial granularity and higher precision are needed in order to improve the identification efficiencies of electrons, photons, tau, jets and missing energy, at high background rejection rates, already at the Level-1 trigger. The LAr Trigger Digitizer system will digitize the 34,000 channels (SuperCells) at a $40 \mathrm{MHz}$ sampling frequency with 12 bit precision after the bipolar shaping of the front-end system. The data will be transmitted to the LAr Digital Processing system in the back-end to extract the transverse energies and perform the bunchcrossing identification. A demonstrator has been installed during Run-2, and the results of the data-taking have helped to validate the chosen technology. Results of ASIC developments including QA/QC and radiation hardness evaluations, performances of the pre-production boards and results of the system integration tests, progress of QA/QC of final production boards will be presented along with the overall system design.

European Physical Society Conference on High Energy Physics - EPS-HEP2019 -

10-17 July, 2019

Ghent, Belgium

${ }^{*}$ Speaker. 


\section{Introduction}

The ATLAS Liquid Argon (LAr) Calorimeters consist of the electromagnetic part which covers the pseudo-rapidity range $0<|\eta|<3.2$ and the hadronic part in the forward region. The instantaneous luminosity will be increased from $L \sim 1.7 \times 10^{34} \mathrm{~cm}^{-2} \mathrm{~s}^{-1}$ for Run-2 (2015-18) to $L \sim 2.2 \times 10^{34} \mathrm{~cm}^{-2} \mathrm{~s}^{-1}$ for Run-3 (2021-23). The Level-1 trigger rate is required to stay less than $100 \mathrm{kHz}$ and, in particular, electromagnetic trigger needs to stay $20 \mathrm{kHz}$.

By the Phase-1 upgrade, the granularity of the trigger readout will be finer to improve the rejection of hadronic jets, one of the background. The trigger readout upto Run-2, called Trigger Towers, is based on an analog sum of signal from the calorimeter cells in the range of $\Delta \eta \times \Delta \phi=0.1 \times 0.1$ for all four layers as shown Figure 1 (a). The trigger readout from Run-3 will be upgraded to Super Cells which are placed for each layer and the granularity is increased by four times with respect to Trigger Towers in the front and middle layer as shown Figure 1 (b). The finer readout in the front and middle layer can reconstruct shower shape better and improve the ability to discriminate between electron/photons and hadronic jets. Therefore, new trigger readout enables the trigger rate to stay $20 \mathrm{kHz}$ without increasing transverse energy threshold as shown Figure 2. The lower threshold of $\sim 25 \mathrm{GeV}$ with Super Cells allows to trigger more events with electrons from $W$ or $Z$ boson decay, also to increase acceptance on events with photons (e.g. $H \rightarrow \gamma \gamma$ ), which are one of the primary targets of the ATLAS physics program.

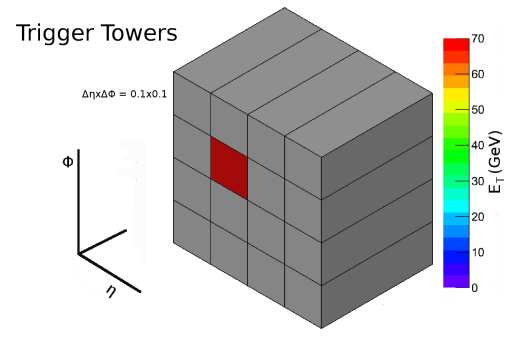

(a) Trigger Towers

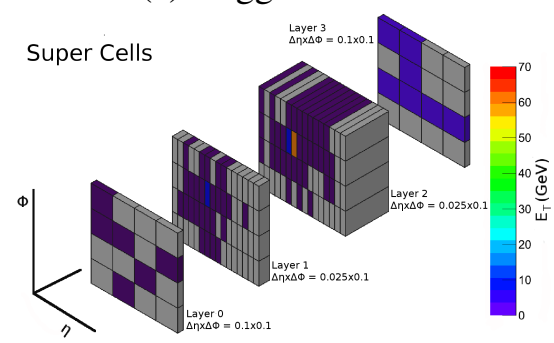

(b) Super Cells

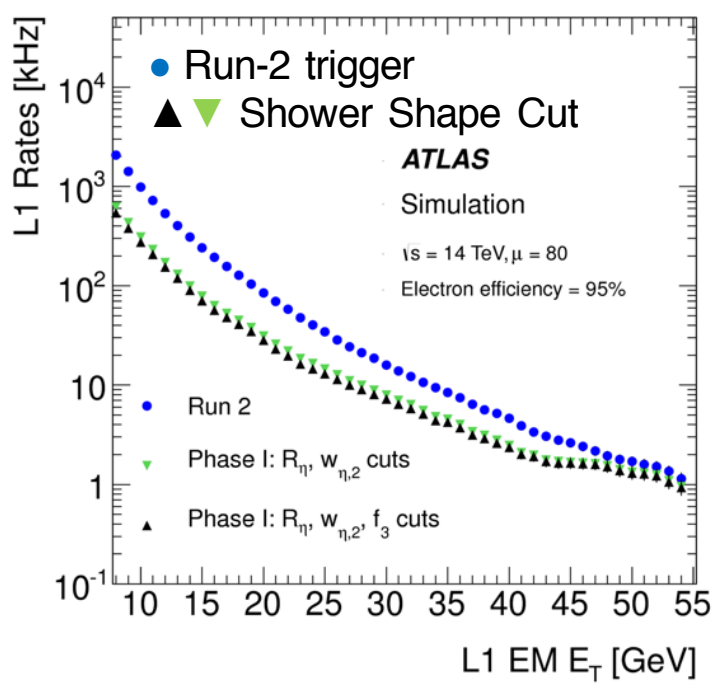

Figure 2: Level-1 trigger rates for a $95 \%$ electron efficiency as a function of threshold on EM $E_{\mathrm{T}}$ for the case of Run 2 conditions (blue points) and Run 3 conditions with shower shape variables (green and black triangles) [1]
Figure 1: An electron with $70 \mathrm{GeV}$ of $E_{\mathrm{T}}$ as seen by Trigger Towers and Super Cells [1] 


\section{New Readout Electronics Components and Production Status}

The current LAr Calorimeter readout architecture remains unchanged, however we need to refurbish some new components in order to realize the new trigger readout with Super Cells in the Phase-1 upgrade (Figure 3). The following components are going to be introduced into the Front-end electronics :

- LAr Trigger Digitizer Board (LTDB) : 124 boards. Digitization of 320 Super Cells signals per board. All custom radiation tolerant ASICs, Quad channel ADC (80 chips per LTDB) which process the analog-to-digital conversion, LOCx2 Serializer (20 per LTDB) and LOCld laser driver (40 per LTDB) which serialize the ADC data for several Super Cells and transmit to the backend system, produced and QC has completed.

- Baseplane : 114 boards. Transmission of increased number of signals to LTDB. Need to be inserted into the LTDB.

- Layer Sum Boards (LSB) : 2456 boards. Mezzanine board in the Front End Board (FEB) which is the main element in Front End electronics. Summing of the signals of the Calorimeter Cells into units of Super Cells. Production was finished.

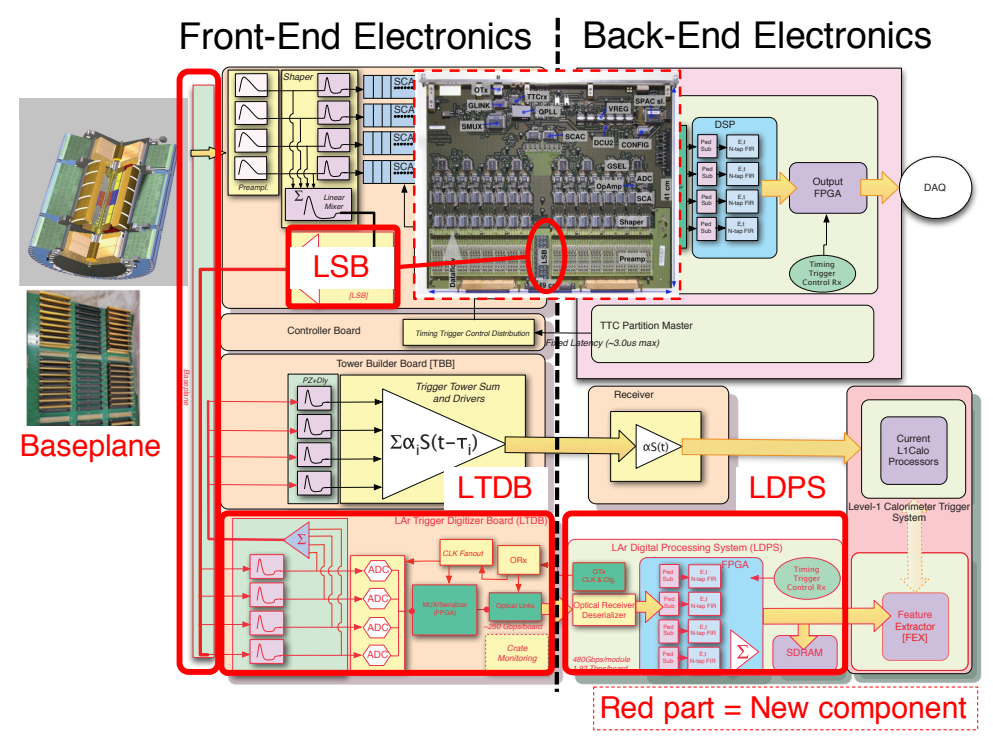

Figure 3: Schematic block diagram of the Phase-1 upgrade LAr trigger readout architecture [1]

For the Back-end electronics, the LAr Digital Processing System (LDPS) which is based on ATCA architecture is going to be introduced. The LDPS reconstructs the transverse energy and bunch-crossing identification and transmits to the Level-1 calorimeter trigger system and is composed two main components :

- LAr Carrier card (LArC): 35 boards, ATCA Carrier blade with RTM card. Fully qualified. 
- LAr Trigger prOcessing MEzzaine (LATOME): 140 boards (4 per LArC). AMC board with Arria-10 FPGA. QC is ongoing.

The photograph of LArC and LATOME is shown in Figure 4. The test of communication between the LArC and LATOME has been completed validation. The improvement of firmware for LATOME has been proceeding and development of the tools for commissioning with new hardware is ongoing. We have succeeded in reading the calibration pulse through LTDB and LATOME as shown in Figure 5.

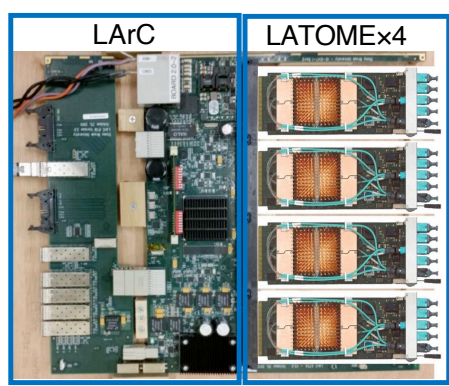

Figure 4: LArC and LATOME

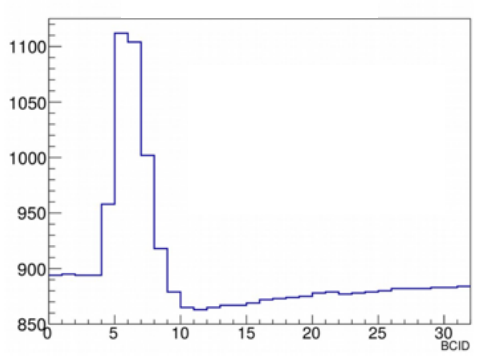

Figure 5: Calibration pulse throgh LTDB and LATOME

\section{Collision data with Demonstrator}

A demonstrator was installed during Run-2. The measured Super Cell energies of the demonstrator are compared to summed LAr cell energies from the main readout.

The event displays from the main readout and the demonstrator are shown in Figure 6. The volume of the boxes is proportional to the deposited energy. Super Cell energies of the LAr Phase-1 demonstrator and summed LAr cell energies are given for the same shower in Figure 6 (a), (b).

The 2D plot of correlation of energy measurements with the demonstrator and with the main readout is shown in Figure 6 (c). Good agreement is observed between the main readout and demonstrator readout.

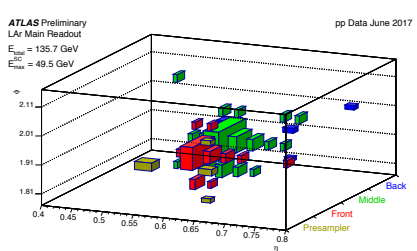

(a) Event display with Main Readout

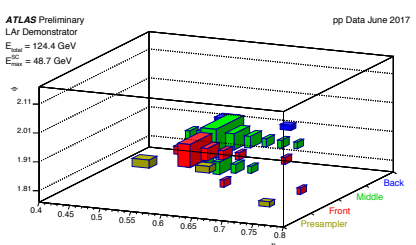

(b) Event Display with Demonstrator

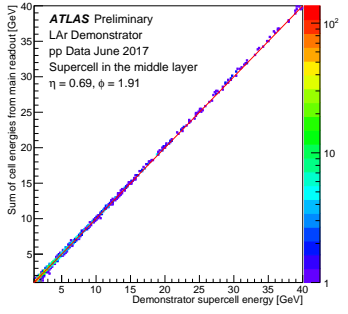

(c) Correlation of energy measurements for middle layer

Figure 6: Event displays with Main Readout (a) and with Demonstrator (b). The 2D plot of correlation of energy measurements with the demonstrator and with the main readout for middle layer (c). [2] 


\section{Installation Status}

The commissioning has begun and we have three systems to commission :

- Main readout : Check refurbished FEBs are connected well and have same performance as before

- Analog trigger path : Check the LTDBs provide correct analog sums to TBBs

- New digital trigger path : Check the full chain readout

Front-End installation is planned to be finished by March 2020. The current situation of FrontEnd installation is that one of the crates (End-Cap A03 Crate) was tested and FEB readout with main data path. The plot of pulse with A03 Crate is shown in Figure 7.

Back-End commissioning is planned for October 2019. The integration with TDAQ and new Timing Trigger Control (TTC) is ongoing.

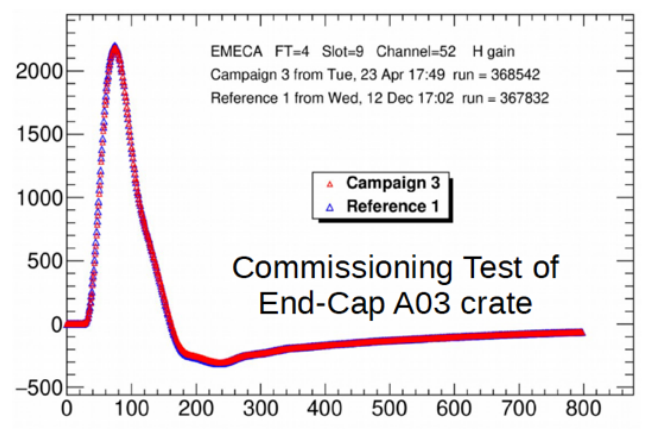

Figure 7: Pulse with End-Cap A03 crate. Good agreement between the reference which is taken during Run-2 (blue points) and the test pulse on commissioning (red points)

\section{Summary}

This paper presents the current status of the production and installation of new electronics for trigger readout on the ATLAS LAr Calorimeter Phase-1 upgrade. The demonstrator which was installed during Run-2 shows that good agreement is observed between the main readout and demonstrator readout. All custom radiation tolerant ASICs on LTDB has been produced and QC has completed. On the Back-End electronics, the development including QA/QC is ongoing and the LDPS firmware finalization is also underway. The commissioning has begun and we succeeded to read the pulse with End-Cap A03 Crate. The installation is planned by October 2019 on the Back-End electronics and by March 2020 on the Front-End electronics.

\section{References}

[1] ATLAS Collaboration, ATLAS Liquid Argon Calorimeter Phase-I Upgrade Technical Design Report, https://cds.cern.ch/record/1602230

[2] Developments of the ATLAS Liquid Argon Calorimeter Phase-I upgrade, ATLAS collaboration, https://twiki.cern.ch/twiki/bin/view/AtlasPublic/LArCaloPublicResultsUpgrade. 